\title{
AI for manufacturing: Some practical experience
}

\author{
J. Lažanský, V. Mařík \\ Czech Technical University, Faculty of Electrical Engineering, \\ Technická 2, CZ-166 27 Prague, Czech Republic \\ Phone: (+42 2) 293107, Fax: (+42 2) 290159 \\ E-mail: \{marik, lazansky\}@lab.felk.cvut.cz
}

\begin{abstract}
The paper gathers authors' experience with industrial applications of AI methods. Many tasks from the area of manufacturing motivate investigations in AI and vice versa. Two particular examples of software systems for computer support for manufacturing in small and medium sized enterprises are discussed. The first series of systems is introduced, and the problem of constrained sequencing of operations is discussed based on the classical problem solving theory. The other example involves operative planning as a discrete optimization task. An attempt to solve this problem using genetic algorithms is introduced and some experimental results are provided. The conclusions summarize authors' observations on AI methods in the design of software for manufacturing.
\end{abstract}

\section{Keywords \\ CIM, artificial intelligence, computer-aided production planning}

\section{INTRODUCTION}

Besides of the individual AI methods and techniques (like state-space search, knowledge representation, uncertainty processing) which are rarely directly applicable in manufacturing, the AI has brought a new philosophy of software development, integration and reusability. This philosophy may be even more important outcome of AI than the individual techniques.

We would like to demonstrate in this paper that the individual AI methods and techniques proved to be also useful and applicable in solving routine industrial tasks. But these methods are usually too general to be directly applied. Their application requires a detailed analysis of the task, followed by an ad hoc design and development of the software in which modified AI techniques and their appropriate combinations are involved in a natural way. 
Two examples of larger software systems for manufacturing are briefly presented in this paper with the goal to summarize some experience concerning the industrial AI applications and to show their impacts on SMEs.

\section{EXAMPLE I: CAPP SYSTEM TEPRO}

The Computer Aided Production Planning (CAPP) systems bridge the gap between CAD and the workshop production needs. These systems are used to transform product definition data which have been generated by CAD into data necessary for the production process. This transformation has to consider many physical constraints given by the manufacturing environment (Bray, 1988). For example: to manufacture a certain part, a sequence of production steps (operations) on various machines has to be done.

\subsection{Manual versus CA planning}

In a classical manufacturing environment the process planner (a person) makes the plan 'by hand' using various tables, having in mind all manufacturing facilities in the particular factory. By tradition, the planner normally makes use of a set of predefined forms with various items about material, working site to be used, textual description of the operations, tools, etc.

A detailed analysis of requirements on CAPP systems has been done in several Czech companies. All these companies were interested in CAPP systems for supporting mainly the logistic problems of the production planning. To illustrate the problem, consider the manufacturing of simple mechanical components. Each of them is produced in a series of operations. Here the 'operation' is considered as any processing done at one working site (machine, workcell), no matter how complicated the processing is.

Various working sites in the workshop differ substantially in their versatility and processing attributes. To choose particular machine, many properties have to be taken into account. These include: capability of the machine to provide the particular type of operations, their effectivity and accuracy, capability to process the given material at the current size, etc.

Moreover, particular machines are not involved in the manufacturing process as isolated units. Since the given product usually cannot be finished on one machine it is often necessary to think about the possibilities of in-process transport which is very often accomplished by making use of in-process buffering stores.

When a machine finishes its operation the product (or a batch of products) is forwarded into the corresponding store. Thus the next operation on the same product can be carried out only on a machine that is accessible from that store. Although the in-process transport system may be organized in another way, it should always be considered as a restriction for the sequence of operations. A graph structure can be used to model the transport system. Nodes of this graph represent single workplaces and the edges play the role of transport connections. This graph is not a complete one thus imposing non-relaxable constraints on the workplace sequence.

The planner is expected to create the plan using the product drawing (or the outputs of a CAD system) and having a general idea on the ordering of necessary operations. The user has to make many decisions during the detailed process plan creation. There are also many routine calculations so that the process plan be full and correct. Many data from various tables and standards have to be filled in corresponding form items. 
The analysis lead to the following conclusion agreed with the customers. The CAPP systems are expected to deliver production plans similar to those prepared manually. This suggested to design the CAPP systems as interactive knowledge-based screen editors of predefined forms. Creating a new production plan can be considered as editing of a dynamically concatenated series of empty forms. Each form is displayed and edited on the terminal screen.

The editor offers and accepts only those user's actions that are in concordance with the knowledge base of the CAPP system. This mechanism of the constraint-directed planning keeps the user within the constraints preserving his/her freedom in decision making. The editor interprets the user's decisions and transfers all of the relevant data from the corresponding data base to the production plan form.

\subsection{State space approach}

From the viewpoint of $\mathrm{AI}$, the task of computer-aided planning may be considered as a planning with constraints in a large state space. The state space reflects possible sequences of operations performed on machines existing in the given manufacturing environment. The process plan forms a trajectory in this space. The knowledge on the manufacturing environment (describing, e.g., capabilities of machines, in-process transport restrictions, etc.) can reduce dramatically the problem solving process in a very large state space.

Let us illustrate the situation of building a linear plan by an example of choosing a suitable workplace (machine). This is one of the most complicated points of the process plan design. The machine has to be capable to carry out the desired operation on given material while current dimensions of the semi-product have to be taken into account, too. Moreover the workplace has to be accessible from that one where previous operation has taken place due to in-process transport system. When all these restrictions are put together, they reduce the set of admissible machines to several ones from original high number of workplaces.

A family of CAPP systems, called TEPRO, developed at the Czech Technical University in the last decade is a group of knowledge-based decision-support systems for complex and flexible manufacturing environments. In these systems, the human operator plays the role of a supervisor. At the beginning of the work, the operator presents the material and a rough sequence of basic (macro-)operations (like cutting, drilling, punching, turning) by use of a set of menus. Next the rough sequence is being refined by assigning the operations to suitable working sites based on predefined textual descriptions of the operations. Now the system offers only acceptable alternatives for each operation by making use of all available information.

The system considers especially machine capabilities, transport flow constraints, material restrictions, dimensional limits, precision requirements, and the machine load statistics. All these components are used to generate a sorted list of admissible working sites on each step of the planning. The user makes the final choice among those alternatives or recommends to consider several of them.

To reuse the results, the authors have decided to find a formal model of the task which is described in (Lažanský, 1995) in more detail.

The AI-based philosophy of the CAPP systems issues from the consideration that the desired sequence of technological operations can be considered as a trajectory in a state space. 
The idea of the state-space search controlled by knowledge seems to be a good solution. But the problem and its formalization is not simple.

Two different, but tightly linked, dual state spaces have been designed for this purpose.

- State space $F_{1}$ of physical manufacturing of real (semi-)product (the states represent the current states $s_{n}$ of the semi-product, the transitions $o_{j}$ correspond to manufacturing operations);

- State space $F_{2}$ of the semi-product transport (this can be described by the topological graph of transport paths with nodes representing individual workplaces $w_{i}$ and transitions $t_{j}$ corresponding to transport links.)

The production planning may be described in the following way. At the very beginning, the user has a rough idea about the first operation to be performed. Thus, the choice of the first operation $\left(o_{1}\right.$ in $\left.F_{1}\right)$ is made. All the acceptable transitions are immediately projected to $F_{2}$, with the restrictions being considered. The user chooses among these acceptable transitions. Thus both the workplace $w_{1}$ and the connection path (in-process store) are determined. Whenever the user chooses the operation $\mathrm{O}_{2}$, all possible transitions in $F_{2}$ are immediately displayed and offered for selection. The user stepwise chooses one of them until the final state $s_{n}$ is reached.

Each step in one state-space is followed by one step in the other space.

The problem-dependent knowledge substantially restricts the acceptable transitions in $F_{2}$ : it deals with knowledge on transport paths, manufacturability of the material and/or semiproduct. restrictions given by dimensions etc. Thus, the state space search in $F_{2}$ is dramatically reduced to one or small numbers of alternatives at each of the steps.

It is quite understandable that the formalization of the state space approach by the two dual spaces is rather complicated. The state space $F_{2}$ describes the topological graph, but the fact that this graph is reduced in each step by imposing knowledge on it justifies the state-space formalization. Moreover, the restrictions put on the planning process cannot be expressed efficiently in the natural state-space of real manufacturing.

\section{Alternatives in plans and replanning}

A very important possible extension of the methodology described above includes alternatives in the plan. In the case the alternative machines are considered, a set $W_{i}=\left\{w_{i}\right\}$ of mutually interchangable workplaces is handled instead of $w_{i}$ in both the state spaces. The possibility to derive alternative plans is very important in the phase of creating the schedules. When a cost is assigned to each of the alternatives, it may be used during the execution planning (scheduling) for the estimation of schedule goodness.

New plans may be derived from already existing and verified plans. This is a very important way of fast and efficient creating of new plans. There are three types of actions which could be performed by the user. It is possible

- to change some parameters of an already existing operation.

- to delete an operation,

- to insert a new operation,

After each change in the current plan, it is necessary to check the global correctness of the new plan, i.e. to test

- whether all preconditions of the inserted or changed operation are satisfied, as well as,

- whether the operations subsequent to the deleted, inserted or changed operation are executable. 
Such a checking is a specific replanning task - it can be considered as an analogy to the truthmaintenance (Sundermeyer, 1991) in a wider sense. It is solved by the simplest and the most efficient way: After each change of a plan, the global checking procedure may be applied to the new, derived plan. This procedure tries to pass successfully through both the state-spaces $F_{1}, F_{2}$ starting from the initial states quite automatically (without any interaction with the user). If some preconditions or restrictions are not satisfied, the procedure stops its activity and informs the user immediately. The plans which were not successfully proved by the checking procedure are prevented from being handled as correct plans (i.e. stored in the list of correct plans, etc.).

\subsection{Implementation of TEPRO systems}

A family of the knowledge-based CAPP systems TEPRO is based on the philosophy described above. The systems have been used in different factories in the Czech Republic.

The TEPRO system is supported by an information base which is split into two parts: data and knowledge. All the necessary facts and the available knowledge is used for decision making support. The data part of the information base consists of the manufacturing data relevant to the scope of the production type. The knowledge expresses the restrictions resulting from particular workshop or factory organization and facilities and corresponds to knowledge of a highly skilled process planner. Moreover the knowledge also describes how to manipulate the data.

The TEPRO-systems have been developed for various computer architectures and for diverse manufacturing environments. As a matter of fact, they are bound together mainly by the common AI planning philosophy.

The first implementation of the TEPRO system was developed for a flexible manufacturing system with about 50 machines for manufacturing mechanical parts of flat material in TESLA Kolín Company.

The system TEPRO-ČKD represents the largest TEPRO implementation covering all the demands on production planning on the whole factory level. It has been developed for the CKDD-TATRA Comp. which is the world's largest manufacturer of trams (street cars) with an important subsidiary in Brazil. Besides manufacturing, it was necessary to capture also the problem of creation of assembling plans which are - in principle - non-linear and very often treelike. Such plans are organized as piecewise linear ones, the 'connection points' being located in the assembling cells.

The TEPRO-ČKD has been directly linked to the huge, already existing data-base of the CKKD Comp. and the information base has been integrated in it.

From the AI point of view, an interesting approach called prototype plans has been introduced. For instance, all the plans for manufacturing doors of different trams are very similar. Prototype plans stored in the database represent 'typical paths' through the state space. When creating a new plan (for manufacturing a little bit different door), it is possible to use the typical plan and to carry out some slight changes in it. The main problem which remains, concerns the proof of admissibility (manufacturability) of the new path in the state space.

The TEPRO-ČKD has been run daily. About 35,000 production plans consisting of nearly 200,000 operations have been created using the system. 


\section{EXAMPLE II: SYSTEM PACEB}

\subsection{Formulation of the task}

As another example, let's present a CIM case-study system called PACEB (an acronym of a Czech company). The PACEB system solves a complete CIM task in a small-sized factory that manufactures building prefabricates. The system includes data management on product assortment, inventory management, reception of orders with the chance of customer specified changes of the standard products, invoicing with payment checking, and many other daily administrative routines.

From the AI point of view, the most interesting part of the system was the demand to solve the tasks of operative planning. The task is highly constrained.

The standard assortment of prefabricates (panels) consists of about 550 types differing in dimensions, shape, construction, material, and edge profile. The factory receives orders containing desired numbers of customer chosen products. The delivery dates are estimated and the orders are to be processed to satisfy the customer requirements. Many technological and managerial constraints have to be taken into account:

(1) Each prefabricate has to harden 28 days before it can be dispatched.

(2) The planning has to consider limited capacity of the factory.

(3) Due dates satisfaction on whole orders without superfluous stocking is highly desirable.

(4) The production operates in 24 hours cycles.

(5) Panels are manufactured on special mobile bases on which boarding is fixed to create the prefabricates shape. The factory possesses bases of 4 different dimensions.

(6) A set of prefabricates is planned for each base to achieve maximal utilization of the base area. Only certain combinations of prefabricates can be scheduled for each base. The admissibility of prefabricates arrangement is stated in terms of their dimensions, edge profile and material composition.

(7) Care must be taken to minimize the necessity of boarding rearrangements on each base compared to 'yesterday' layout. This is a soft constraint.

By a deeper analysis, the conditions can be split into two groups: conditions involving temporal dependencies $(1,2,3,4)$ and constraints of geometric nature $(5,6,7)$. The first group of conditions can be used to form a priority queue of panels waiting for manufacture.

The interesting part of the problem is the task of planning the prefabricates from a queue onto the production bases. This problem is a typical task of discrete optimization. The objective function can be formulated as a combination of two antagonistic requirements: maximization of daily profit and minimization of the boarding rearrangement. The constraints for this task can be hardly expressed analytically.

\subsection{Discrete optimization using genetic algorithms}

We have decided to test the applicability of genetic algorithms (GA) in this optimization task. GA represent a very promising $\mathrm{AI}$ technique based on simulation of evolution in nature.

Our first experiments with GA applications for classical optimization problems were very encouraging. Thus, we decided to use this approach for a simplified version of the task described in the previous section. 
The crucial problem of the GA application for a constrained optimization task is the problem of representation. The question is how to represent the points of the search space so that reasonably simple and efficient genetic operators can be used. Simply represented discrete problems generate a huge majority of candidate solutions that are infeasible. For constrained cases many sophisticated ad hoc representations and corresponding genetic operators have been proposed. Their aim is to reduce the ratio between the number potentially generated candidate solutions and that of feasible ones. Some of those methods can be found in (Michalewicz, 1992).

The GA have a very important property: They can run infinitely in a good hope of finding a yet better solution. The best-so-far solution cannot get lost.

\subsection{The GA-based approach}

The first testing task was formulated for one-dimensional case as a problem of cutting a rod of length $L$. It is necessary to cut the rod into short pieces of various lengths $l_{i}$ and given number $n_{i}$ of each of them. The objective is to minimize the waste. The task can be considered as a one-dimensional knapsack problem. The experimental results have been surprisingly good and their detailed description can be found in (Kouba, 1994).

The results encouraged further extension to two dimensions with constraints very close to those in the original PACEB system. To simplify the GA task, some preprocessing of the queued panels has been done. The panels have been grouped so that prefabricates that can fit in a row on the mobile base are in one group (cf. condition (6) in the previous paragraph). A separate GA run was taken for each group, forming some possible rows arrangements. In the next step. the previously obtained rows were combined using GA to achieve maximum coverage of the mobile bases area.

For an experimental evaluation, a typical set of panels was chosen and the coverage of mobile bases was computed by a very time-consuming branch-and-bound method. Than, the briefly described two steps GA was run 10 times. In 9 of those runs, the optimum was achieved in less than $20 \%$ of the time needed for the branch-and-bound computation.

\section{CONCLUSIONS}

Two examples of the AI-based solutions aimed to support mainly the SME manufacturers in the Czech Republic have been presented. A lot of experience has been gathered during both the development and exploration phases of these solutions.

1. Every application of AI ideas requires a very deep analysis of the industrial problem to be solved. This analysis typically brings new information to the manufacturer and helps him to recognize, define and understand the information flows in the production environment. As a consequence, some changes in the organization of production and supporting activities can be immediately accomplished by the company.

2. Nearly all the solutions based on the requirements analysis lead to knowledge-based structures and control algorithms designed ad hoc. This is partially true also for implementation. All of the TEPRO implementations (even though based on the same philosophy) always required a new environment analysis which resulted in substantial changes in architecture and knowledge representation formalisms, which finally lead to programming from scratch. The requirement of software re-usability in the CIM area leads 
to multi-agent solutions based on the object-oriented programming. Real solutions based on the ideas and technology of an agent factory (O'Hare, 1993) are still far from being widely applicable in industry.

3. The problem-oriented, environment specific solutions are typically based on some AI principles which should be modified, combined and extended in a creative way. The creativity is used mainly for transforming and re-formulating the problem into a form enabling the use of some AI (even modified or combined) algorithms. Standardized prototype solutions making the transformation problem easier are not available.

4. As the software solutions are very expensive, the manufacturers are introducing their CIM software step by step: The projects usually cover only a part of the global CIM visions. This fact imposes new constraints on the solutions: (1) They should be compatible with the 'rest' of the company activities and (2) they should respect the already existing traditions, habits and style of work in the company.

5. The management on all factory levels prefer transparent and understandable software solutions which offer acceptable solutions leaving the decision itself at a human operator. By our experience: A fully automated decision making is rarely appreciated and hardly acceptable by the end-user.

6. The software developers are welcome to use the latest AI technology. The use of up-to-date (and from the AI viewpoint attractive) terminology is not recommended even if the corresponding technology is being used. The people in industry do not trust these methods which they consider as too difficult to understand. This fact could bring an artificial barrier between the $\mathrm{AI}$ system designers and end-users (having, hopefully, natural intelligence).

\section{REFERENCES}

Bray, O. H. (1988) CIM: The data management strategy. Digital Press, New York

Kouba, Z., Lažanský, J., Mařík, V., Vlček, T., Ženíšek, P. (1994) Experiments with Genetic Algorithms in a CIM Task, in: Cybernetics and Systems Research (ed. R. Trappl), World Scientific Publ., Singapore, Vol. 2, pp. 1833-1841

Lažanský, J., Kouba, Z., Mařik, V., Vlček, T. (1995) Production Planning Systems with AI Philosophy. Int. Journal on Expert Systems with Applications, Elsevier Science, Vol. 8, No. 2, pp. 255-262

Mařik, V., Lažanský, J. (1994) Applying AI for the Development of CIM Software, in: Proceedings of 13th World Computer IFIP Congress 94 (ed. K. Duncan and K. Kruger), Elsevier Science, Vol. 3, 1994, pp. 416-423

Michalewicz Z. (1992) Genetic Algorithms + Data Structures = Evolution Programs, Springer-Verlag, Berlin

O'Hare, G.M.P. and Wooldridge, M.J. (1993) A Software Engineering Perspective on Multi-Agent System Design, in Distributed AI: Theory and Praxis (ed. M.N. Avouris, L. Gasser), Kluwer Academic Publ., Dordrecht, pp. 109-128

Sundermeyer. K. (1991) Knowledge-based Systems. BI Wissenschaftsverlag, Mannheim, Germany 